\title{
An Averaging Principle for Caputo Fractional Stochastic Differential Equations with Compensated Poisson Random Measure
}

\author{
GUO Zhongkai ${ }^{1}$, FU Hongbo ${ }^{2}$ and WANG Wenya ${ }^{3, *}$ \\ 1 School of Mathematics and Statistics, South-Central University for Nationalities, \\ Wuhan 430074, China. \\ 2 Research Center of Nonlinear Science, College of Mathematics and Computer \\ Science, Wuhan Textile University, Wuhan 430074, China. \\ ${ }^{3}$ School of Artificial Intelligence, Jianghan University, Wuhan 430056, China.
}

Received 13 September 2020; Accepted 2 May 2021

\begin{abstract}
This article deals with an averaging principle for Caputo fractional stochastic differential equations with compensated Poisson random measure. The main contribution of this article is impose some new averaging conditions to deal with the averaging principle for Caputo fractional stochastic differential equations. Under these conditions, the solution to a Caputo fractional stochastic differential system can be approximated by that of a corresponding averaging equation in the sense of mean square.
\end{abstract}

AMS Subject Classifications: 34C29, 39A50

Chinese Library Classifications: O211

Key Words: Stochastic fractional differential equations; averaging principle; compensated Poisson random measure.

\section{Introduction}

Most systems in science and industry are perturbed by some random environmental effects, described by stochastic differential equations with (fractional) Brownian motion, Lévy process, Poisson process and etc. A series of useful theories and methods have been proposed to explore stochastic differential equations, such as invariant manifolds, averaging principle, homogenization principle. All of these theories and methods develop to extract an effective dynamics from these stochastic differential equations, which

*Corresponding author. Email addresses: zkguo@scuec.edu.cn (Z. K. Guo), hbfu@wtu.edu.cn (H. B. Fu), Wenyawang2014@qq. com (W. Y. Wang) 
is more effective for analysis and simulation. For averaging principle, its often used to approximate dynamical systems with random fluctuations, and provides a powerful tool for simplifying nonlinear dynamical systems. The essence of averaging principle is to establish an approximation theorem that a simplified stochastic differential equation is presented to replace the original one in some senses.

The averaging principle for stochastic differential equations was first introduced by Khasminskii in paper [1], which extending the deterministic result of [2]. Since then, the theory of averaging principle for stochastic differential equations driven by different noise are considered by many authors, see [3-7] .

Because the non-local property of time derivatives, the model of Caputo fractional stochastic differential equations applied in many areas, such as biology, physics and chemistry and etc.. Existence and uniqueness of solution for Caputo fractional stochastic differential have been discussed by many papers. Quite recently, some types of Caputo fractional stochastic (partial) differential equations problem are considered from the dynamic viewpoint. For example, in paper [8], existence of stable manifolds is established. In [9], the existence of global forward attracting set for stochastic lattice systems with a Caputo fractional time derivative in the weak mean-square topology is considered. The asymptotic distance between two distinct solutions under a temporally weighted norm is discussed by [10]. To the best of our knowledge, averaging principle for Captuo fractional differential equations only considered by two articles in present, see [11] and [12], it is worth noting that the average conditions given in these two papers are different, under this conditions, the corresponding averaging conclusions are drawn respectively. In this paper, we also impose a new averaging condition for our framework, which let us to derive the averaging principle for our consider problem from the theoretical derivation.

Let $\left(\Omega, \mathcal{F}, \mathcal{F}_{t}, \mathbb{P}\right)$ be a complete stochastic base. Let $(Z, \mathcal{B}(Z))$ be a measurable space and $v(d z)$ a $\sigma$-finite measure on it. Let $p=(p(t)), t \in D_{p}$, be a stationary $\mathcal{F}_{t}$-Poisson point process on $Z$ with characteristic measure $v$. The counting measure associated with $p(t)$ is given by, for $A \in \mathcal{B}(Z), N((0, t], A):=\sharp\left\{t \in D_{p}: 0<s \leq t, p(s) \in A\right\}$. Assume that $b: \mathbb{R}_{+} \times \mathbb{R}^{d} \rightarrow \mathbb{R}^{d}, \sigma: \mathbb{R}_{+} \times \mathbb{R}^{d} \rightarrow \mathbb{R}^{d} \times \mathbb{R}^{m}$ and $F: \mathbb{R}_{+} \times \mathbb{R}^{d} \times Z \rightarrow \mathbb{R}^{d}$ are measurable functions. For $\alpha \in\left(\frac{1}{2}, 1\right)$, we consider a stochastic fractional differential equation with compensated Poisson random measure of the form:

$$
D_{t}^{\alpha} X_{\epsilon}(t)=\epsilon b\left(t, X_{\epsilon}(t-)\right) \mathrm{d} t+\sqrt{\epsilon} \sigma\left(t, X_{\epsilon}(t-)\right) \mathrm{d} B_{s}+\sqrt{\epsilon} \int_{Z} F\left(t, X_{\epsilon}(t-), z\right) \tilde{N}(\mathrm{~d} t, \mathrm{~d} z),
$$

where $\epsilon$ is a small positive parameter, $\tilde{N}(\mathrm{~d} t, \mathrm{~d} z):=N(\mathrm{~d} t, \mathrm{~d} z)-\mathrm{d} t v(\mathrm{~d} z)$ is the compensated Poisson martingale measures corresponding to $N(\mathrm{~d} t, \mathrm{~d} z)$ and $\left\{B_{t}\right\}_{t \geq 0}$ is an $m$-dimensional standard $\mathcal{F}_{t}$-adapted Brownian motion. We note that the above equation is a classical equation if $\alpha=1$, which has been studied by many authors. Under some conditions, which can be compared with the classic case as in [12], we derive an averaging principle for the stochastic fractional differential system (1.1).

This article is organized as follows. In Section 2 we will give some assumptions and basic results for our theory. The solution of convergence in mean square between the 
stochastic fractional differential equations with Poisson random measure and the corresponding averaged equation are considered in Section 3.

Throughout this paper, the letter $C$ below will denote positive constants whose value may change in different occasions. We will write the dependence of constant on parameters explicitly if it is essential.

\section{Stochastic differential equations with Poisson random mea- sure}

\subsection{Basic Hypothesis and some useful results}

We impose the following assumptions to guarantee the existence of the solution.

H1 (Lipschitz condition). There exists a bounded function $K_{1}(t)>0$ such that for all $x, y \in R^{d}$,

$$
\begin{aligned}
& |b(t, x)-b(t, y)|^{2}+|\sigma(t, x)-\sigma(t, y)|^{2}+\int_{Z}|F(s, x, z)-F(s, y, z)|^{2} v(\mathrm{~d} z) \\
\leq & K_{1}(t)\left(|x-y|^{2}\right) .
\end{aligned}
$$

H2 (Growth condition). There exists a bounded function $K_{2}(t)>0$ such that for all $x \in R^{d}$,

$$
|b(t, x)|^{2}+|\sigma(t, x)|^{2}+\int_{Z}|F(t, x, z)|^{2} v(\mathrm{~d} z) \leq K_{2}(t)\left(1+|x|^{2}\right) .
$$

We also assume that the $K_{i}(t)$ have the same upper bound $K$, for $i=1,2$.

To deal with fractional differential equation, we need the following generalization of Gronwall's lemma for singular kernels [13,14].

Lemma 2.1. Suppose that $b \geq 0$ and $\beta>0$. Assume that $a(t)$ and $u(t)$ are nonnegative and locally integrable functions on $0 \leq t<T$, satisfying

$$
u(t) \leq a(t)+b \int_{0}^{t}(t-s)^{\beta-1} u(s) \mathrm{d} s .
$$

Then

$$
u(t) \leq a(t)+\int_{0}^{t}\left[\sum_{n=1}^{\infty} \frac{(b \Gamma(\beta))^{n}}{\Gamma(n \beta)}(t-s)^{n \beta-1} a(s)\right] \mathrm{d} s, \quad 0 \leq t<T .
$$

\subsection{Existence and Uniqueness}

In this part, we consider the existence and uniquness for the following equation under conditions $\mathrm{H} 1$ and $\mathrm{H} 2$ :

$$
D_{t}^{\alpha} X(t)=b(t, X(t-)) \mathrm{d} t+\sigma(t, X(t-)) \mathrm{d} B_{t}+\int_{Z} F(t, X(t-), z) \tilde{N}(\mathrm{~d} t, \mathrm{~d} z)
$$


Definition 2.1. A map $X(t):[0, T] \rightarrow L^{2}\left(\Omega, \mathbb{R}^{d}\right)$ is called a solution of the initial value problem (2.1) if $X(0)=x_{0}$ and $X(t)$ is càdlàg and satisfies for $t \in[0, T]$,

$$
\begin{aligned}
X(t)=x_{0}+ & \frac{1}{\Gamma(\alpha)} \int_{0}^{t}(t-s)^{\alpha-1} b(s, X(s-)) \mathrm{d} s+\frac{1}{\Gamma(\alpha)} \int_{0}^{t}(t-s)^{\alpha-1} \sigma(s, X(s-)) \mathrm{d} B_{s} \\
& +\frac{1}{\Gamma(\alpha)} \int_{0}^{t} \int_{Z}(t-s)^{\alpha-1} F(s, X(s-), z) \tilde{N}(\mathrm{~d} s, \mathrm{~d} z) .
\end{aligned}
$$

Using similarly methods as [15], we can derive the global existence and uniqueness of solutions for Eq.2.1. Similar problem also considered by [13] under different framework. In the following, we just given a priori estimate for the solution $X(t)$, which assure the existence of workspace for our problem.

Theorem 2.1. Under conditions of $\mathbf{H} 1$ and $\mathbf{H 2}$, for every $x_{0} \in L^{2}\left(\Omega, \mathbb{R}^{d}\right)$ there exists a unique solution to Eq. (2.2), such that

$$
\sup _{0 \leq t \leq T} E|X(t)|^{2}<\infty
$$

Proof. The existence and uniqueness can be easily proved by the contraction mapping argument and hence omitted here. Next, we estimate the solution $X(t)$ in $L^{\infty}\left([0, T], L^{2}(\Omega\right.$; $\left.\mathbb{R}^{d}\right)$ ). By employing the simple arithmetic inequality

$$
|a+b+c+d|^{2} \leq 4\left(|a|^{2}+|b|^{2}+|c|^{2}+|d|^{2}\right),
$$

we have

$$
\begin{aligned}
E|X(t)|^{2} \leq 4 E\left|x_{0}\right|^{2}+4 E\left|\frac{1}{\Gamma(\alpha)} \int_{0}^{t}(t-s)^{\alpha-1} b(s, X(s-)) \mathrm{d} s\right|^{2} \\
+4 E\left|\frac{1}{\Gamma(\alpha)} \int_{0}^{t}(t-s)^{\alpha-1} \sigma(t, X(s-)) \mathrm{d} B_{s}\right|^{2} \\
+4 E\left|\frac{1}{\Gamma(\alpha)} \int_{0}^{t} \int_{Z}(t-s)^{\alpha-1} F(s, X(s-), x) \tilde{N}(\mathrm{~d} s, \mathrm{~d} z)\right|^{2} \\
=: 4 I_{1}+4 I_{2}+4 I_{3}+4 I_{4} .
\end{aligned}
$$

For $I_{2}$, by Cauchy-Schwarz inequality and the condition $\mathbf{H 2}$, we have

$$
\begin{aligned}
I_{2} & \leq \frac{T K}{\Gamma(\alpha)^{2}} \int_{0}^{t}(t-s)^{2(\alpha-1)}\left(1+E|X(s)|^{2}\right) \mathrm{d} s \\
& \leq \frac{T K}{\Gamma(\alpha)^{2}}\left[\frac{t^{2 \alpha-1}}{2 \alpha-1}+\int_{0}^{t}(t-s)^{2(\alpha-1)} E|X(s)|^{2} \mathrm{~d} s\right] \\
& \leq \frac{K}{\Gamma(\alpha)^{2}} \frac{T^{2 \alpha}}{2 \alpha-1}+\frac{T K}{\Gamma(\alpha)^{2}} \int_{0}^{t}(t-s)^{2(\alpha-1)} E|X(s)|^{2} \mathrm{~d} s,
\end{aligned}
$$

where we have used the fact that $\{s: X(s) \neq X(s-)\}$ is countable. 
For $I_{3}$, by Itô isometry and the condition $\mathbf{H 2}$, we have

$$
\begin{aligned}
I_{3} & \leq \frac{K}{\Gamma(\alpha)^{2}} \int_{0}^{t}(t-s)^{2(\alpha-1)}\left(1+E|X(s)|^{2}\right) \mathrm{d} s \\
& \leq \frac{K}{\Gamma(\alpha)^{2}} \frac{T^{2 \alpha-1}}{2 \alpha-1}+\frac{K}{\Gamma(\alpha)^{2}} \int_{0}^{t}(t-s)^{2(\alpha-1)} E|X(s)|^{2} \mathrm{~d} s .
\end{aligned}
$$

In a similar way, for $I_{4}$ we have

$$
\begin{aligned}
I_{4} & =\frac{1}{\Gamma(\alpha)^{2}} \int_{0}^{t} \int_{|x|<c}(t-s)^{2(\alpha-1)} E|F(t, X(s-), z)|^{2} v(\mathrm{~d} x) \mathrm{d} s \\
& \leq \frac{K}{\Gamma(\alpha)^{2}} \frac{T^{2 \alpha-1}}{2 \alpha-1}+\frac{K}{\Gamma(\alpha)^{2}} \int_{0}^{t}(t-s)^{2(\alpha-1)} E|X(s)|^{2} \mathrm{~d} s .
\end{aligned}
$$

Therefore, we get

$$
E|X(t)|^{2} \leq\left(4 E\left|x_{0}\right|^{2}+\frac{4 K}{\Gamma(\alpha)^{2}} \frac{T^{2 \alpha-1}}{2 \alpha-1}(2+T)\right)+\frac{4 T K+8 K}{\Gamma(\alpha)^{2}} \int_{0}^{t}(t-s)^{(2 \alpha-1)-1} E|X(s)|^{2} \mathrm{~d} s .
$$

By setting $r=4 E\left|x_{0}\right|^{2}+\frac{4 K}{\Gamma(\alpha)^{2}} \frac{T^{2 \alpha-1}}{2 \alpha-1}(2+T)$ and applying Lemma 2.1, we have

$$
\begin{aligned}
E|X(t)|^{2} & \leq r^{2}\left(1+\int_{0}^{t} \sum_{n=1}^{\infty} \frac{\left(\frac{4 T K+8 K}{\Gamma(\alpha)^{2}} \Gamma(2 \alpha-1)\right)^{n}}{\Gamma(2 n \alpha-n)}(t-s)^{n(2 \alpha-1)-1} \mathrm{~d} s\right) \\
& \leq r^{2}\left(1+\sum_{n=1}^{\infty} \frac{\left(\frac{4 T K+8 K}{\Gamma(\alpha)^{2}} \Gamma(2 \alpha-1) T^{2 \alpha-1}\right)^{n}}{\Gamma(2 n \alpha-n+1)}\right) \\
& =r^{2}\left(1+E_{2 \alpha-1,1}\left(\frac{4 T K+8 K}{\Gamma(\alpha)^{2}} \Gamma(2 \alpha-1) T^{2 \alpha-1}\right)\right)<\infty
\end{aligned}
$$

for all $t \in[0, T]$, where $E_{2 \alpha-1,1}(\cdot)$ is a two-parameter function of the Mittag-Leffler type [13]. Then we have

$$
\sup _{0 \leq t \leq T} E|X(t)|^{2}<\infty
$$

This completes the proof of the theorem.

\section{An averaging principle}

We now study an averaging principle for a standard stochastic integral equation in $R^{d}$ :

$$
\begin{aligned}
X_{\epsilon}(t)=x_{0}+ & \frac{\epsilon}{\Gamma(\alpha)} \int_{0}^{t}(t-s)^{\alpha-1} b\left(s, X_{\epsilon}(s-)\right) \mathrm{d} s+\frac{\sqrt{\epsilon}}{\Gamma(\alpha)} \int_{0}^{t}(t-s)^{\alpha-1} \sigma\left(s, X_{\epsilon}(s-)\right) \mathrm{d} B_{s} \\
& +\frac{\sqrt{\epsilon}}{\Gamma(\alpha)} \int_{0}^{t} \int_{Z}(t-s)^{\alpha-1} F\left(t, X_{\epsilon}(s-), z\right) \tilde{N}(\mathrm{~d} s, \mathrm{~d} z),
\end{aligned}
$$


where $x_{0}$ is a random vector satisfy $E\left|x_{0}\right|^{2}<\infty$, and $\epsilon \in\left(0, \epsilon_{0}\right]$ is a positive small parameter with $\epsilon_{0}$ a fixed number.

To ensures an averaging principle, we need to assume the following condition.

H3: There exist measurable functions $\bar{b}: \mathbb{R}^{d} \rightarrow \mathbb{R}^{d}, \bar{\sigma}: \mathbb{R}^{d} \rightarrow \mathbb{R}^{d} \times \mathbb{R}^{m}$ and $\bar{F}: \mathbb{R}^{d} \times Z \rightarrow \mathbb{R}^{d}$ such that for all $t>0$,

$$
\begin{aligned}
& \frac{1}{t^{2 \alpha-1}} \int_{0}^{t}(t-s)^{2(\alpha-1)}|b(s, x)-\bar{b}(x)|^{2} \mathrm{~d} s \leq \varphi_{1}(t)\left(1+|x|^{2}\right), \\
& \frac{1}{t^{2 \alpha-1}} \int_{0}^{t}(t-s)^{2(\alpha-1)}|\sigma(s, x)-\bar{\sigma}(x)|^{2} \mathrm{~d} s \leq \varphi_{2}(t)\left(1+|x|^{2}\right), \\
& \frac{1}{t^{2 \alpha-1}} \int_{0}^{t}(t-s)^{2(\alpha-1)} \int_{Z}|F(s, x, z)-\bar{F}(x, z)|^{2} v(\mathrm{~d} z) \mathrm{d} s \leq \varphi_{3}(t)\left(1+|x|^{2}\right),
\end{aligned}
$$

where $\varphi_{i}(t)$ are positive bounded function with $\lim _{t \rightarrow \infty} \varphi_{i}(t)=0$ for $i=1,2,3$.

Remark 3.1. Note that, when we take $\alpha=1$, the above conditions are consistent with classic case.

Our main result, Theorem 3.1, states that the solution of original equation (3.1) is well approximated, in the sense of mean square, by that of following equation

$$
\begin{aligned}
Z_{\epsilon}(t)=x_{0}+ & \frac{\epsilon}{\Gamma(\alpha)} \int_{0}^{t}(t-s)^{\alpha-1} \bar{b}\left(Z_{\epsilon}(s-)\right) \mathrm{d} s+\frac{\sqrt{\epsilon}}{\Gamma(\alpha)} \int_{0}^{t}(t-s)^{\alpha-1} \bar{\sigma}\left(Z_{\epsilon}(s-)\right) \mathrm{d} B_{s} \\
& +\frac{\sqrt{\epsilon}}{\Gamma(\alpha)} \int_{0}^{t} \int_{Z}(t-s)^{\alpha-1} \bar{F}\left(Z_{\epsilon}(s-), z\right) \tilde{N}(\mathrm{~d} s, \mathrm{~d} z) .
\end{aligned}
$$

Theorem 3.1. Assume Hypotheses H1-H3. Then, for arbitrary $\alpha \in\left(\frac{1}{2}, 1\right), L>0$ and $\beta \in$ $(0,2 \alpha-1)$, there exists a constant $C_{L, \alpha}$ independent of $\epsilon$, such that that

$$
\sup _{0 \leq t \leq L \epsilon^{\frac{-\beta}{2 \alpha-1}}} E\left|X_{\epsilon}(t)-Z_{\epsilon}(t)\right|^{2} \leq C_{L, \alpha} \epsilon^{1-\beta} .
$$

Proof. Let $[0, T]$ be a fixed time interval. For any $t \in[0, T]$ we have

$$
\begin{aligned}
X_{\epsilon}(t)-Z_{\epsilon}(t)=\frac{\epsilon}{\Gamma(\alpha)} & \int_{0}^{t}(t-s)^{\alpha-1}\left[b\left(s, X_{\epsilon}(s-)\right)-\bar{b}\left(Z_{\epsilon}(s-)\right] \mathrm{d} s\right. \\
& +\frac{\sqrt{\epsilon}}{\Gamma(\alpha)} \int_{0}^{t}(t-s)^{\alpha-1}\left[\sigma\left(s, X_{\epsilon}(s-)\right)-\bar{\sigma}\left(Z_{\epsilon}(s-)\right] \mathrm{d} B_{s}\right. \\
& +\frac{\sqrt{\epsilon}}{\Gamma(\alpha)} \int_{0}^{t} \int_{Z}(t-s)^{\alpha-1}\left[F\left(s, X_{\epsilon}(s-), z\right)-\bar{F}\left(Z_{\epsilon}(s-), z\right)\right] \tilde{N}(\mathrm{~d} s, \mathrm{~d} z) .
\end{aligned}
$$


Directly, we get

$$
\begin{aligned}
& E\left|X_{\epsilon}(t)-Z_{\epsilon}(t)\right|^{2} \\
& \leq 3\left\{E\left|\frac{\epsilon}{\Gamma(\alpha)} \int_{0}^{t}(t-s)^{\alpha-1}\left[b\left(s, X_{\epsilon}(s-)\right)-\bar{b}\left(Z_{\epsilon}(s-)\right)\right] \mathrm{d} s\right|^{2}\right. \\
& \quad+E\left|\frac{\sqrt{\epsilon}}{\Gamma(\alpha)} \int_{0}^{t}(t-s)^{\alpha-1}\left[\sigma\left(s, X_{\epsilon}(s-)\right)-\bar{\sigma}\left(Z_{\epsilon}(s-)\right)\right] \mathrm{d} B_{s}\right|^{2} \\
& \left.\quad+E\left|\frac{\sqrt{\epsilon}}{\Gamma(\alpha)} \int_{0}^{t} \int_{Z}(t-s)^{\alpha-1}\left[F\left(s, X_{\epsilon}(s-), z\right)-\bar{F}\left(Z_{\epsilon}(s-), z\right)\right] \tilde{N}(\mathrm{~d} s, \mathrm{~d} z)\right|^{2}\right\} \\
& =: 3\left(J_{1}+J_{2}+J_{3}\right) .
\end{aligned}
$$

For $J_{1}$, we have

$$
\begin{aligned}
J_{1}=E\left|\frac{\epsilon}{\Gamma(\alpha)} \int_{0}^{t}(t-s)^{\alpha-1}\left[b\left(s, X_{\epsilon}(s-)\right)-\bar{b}\left(Z_{\epsilon}(s-)\right)\right] \mathrm{d} s\right|^{2} \\
\leq \frac{2 \epsilon^{2}}{\Gamma(\alpha)^{2}}\left\{E\left|\int_{0}^{t}(t-s)^{\alpha-1}\left[b\left(s, X_{\epsilon}(s-)\right)-b\left(s, Z_{\epsilon}(s-)\right)\right] \mathrm{d} s\right|^{2}\right. \\
\left.\quad+E\left|\int_{0}^{t}(t-s)^{\alpha-1}\left[b\left(s, Z_{\epsilon}(s-)\right)-\bar{b}\left(Z_{\epsilon}(s-)\right)\right] \mathrm{d} s\right|^{2}\right\} .
\end{aligned}
$$

Due to the Cauchy-Schwarz inequality, conditions H1 and (3.2) in H3, we get

$$
\begin{aligned}
J_{1} \leq & \frac{2 t K \epsilon^{2}}{\Gamma(\alpha)^{2}} \int_{0}^{t}(t-s)^{2(\alpha-1)} E\left|X_{\epsilon}(s-)-Z_{\epsilon}(s-)\right|^{2} \mathrm{~d} s \\
& +\frac{2 K \epsilon^{2}}{\Gamma(\alpha)^{2}} t^{2 \alpha}\left\{\frac{1}{t^{2 \alpha-1}} E \int_{0}^{t}(t-s)^{2 \alpha-2}\left|b(s, Z(s-))-\bar{b}\left(Z_{\epsilon}(s-)\right)\right|^{2} \mathrm{~d} s\right\} \\
& \leq \frac{2 t K \epsilon^{2}}{\Gamma(\alpha)^{2}} \int_{0}^{t}(t-s)^{2(\alpha-1)} E\left|X_{\epsilon}(s-)-Z_{\epsilon}(s-)\right|^{2} \mathrm{~d} s+\frac{2 K \epsilon^{2}}{\Gamma(\alpha)^{2}} t^{2 \alpha} \varphi_{1}(t)\left(1+E\left|Z_{\epsilon}(s-)\right|^{2}\right) .
\end{aligned}
$$

For $J_{2}$, using the Itô isometry, we have

$$
\begin{aligned}
J_{2}= & \frac{\epsilon}{\Gamma(\alpha)^{2}} \int_{0}^{t}(t-s)^{2(\alpha-1)} E\left|\sigma\left(s, X_{\epsilon}(s-)\right)-\bar{\sigma}\left(Z_{\epsilon}(s-)\right)\right|^{2} \mathrm{~d} s \\
\leq \frac{2 \epsilon}{\Gamma(\alpha)^{2}} & \left\{\int_{0}^{t}(t-s)^{2(\alpha-1)} E\left|\sigma\left(s, X_{\epsilon}(s-)\right)-\sigma\left(s, Z_{\epsilon}(s-)\right)\right|^{2} \mathrm{~d} s\right. \\
& \left.\quad+\int_{0}^{t}(t-s)^{2(\alpha-1)} E\left|\sigma\left(s, Z_{\epsilon}(s-)\right)-\bar{\sigma}\left(Z_{\epsilon}(s-)\right)\right|^{2} \mathrm{~d} s\right\} .
\end{aligned}
$$


By conditions $\mathbf{H 1}$ and (3.3) in $\mathbf{H 3}$, we obtain

$$
\begin{aligned}
J_{2} \leq \frac{2 \epsilon}{\Gamma(\alpha)^{2}} & \left\{\int_{0}^{t}(t-s)^{2(\alpha-1)} E\left|\sigma\left(s, X_{\epsilon}(s-)\right)-\sigma\left(s, Z_{\epsilon}(s-)\right)\right|^{2} \mathrm{~d} s\right. \\
& \left.+\int_{0}^{t}(t-s)^{2(\alpha-1)} E\left|\sigma\left(s, Z_{\epsilon}(s-)\right)-\bar{\sigma}\left(Z_{\epsilon}(s-)\right)\right|^{2} \mathrm{~d} s\right\} \\
\leq & \frac{2 K \epsilon}{\Gamma(\alpha)^{2}} \int_{0}^{t}(t-s)^{2(\alpha-1)} E\left|X_{\epsilon}(s-)-Z_{\epsilon}(s-)\right|^{2} \mathrm{~d} s \\
& +\frac{2 K \epsilon}{\Gamma(\alpha)^{2}} t^{2 \alpha-1} \varphi_{2}(t)\left(1+E\left|Z_{\epsilon}(s-)\right|^{2}\right) .
\end{aligned}
$$

Finally, we take estimate on $J_{3}$. Using the Itô isometry yields

$$
\begin{gathered}
J_{3}=E\left|\frac{\sqrt{\epsilon}}{\Gamma(\alpha)} \int_{0}^{t} \int_{|x|<c}(t-s)^{\alpha-1}\left[F\left(t, X_{\epsilon}(s-), z\right)-\bar{F}\left(Z_{\epsilon}(s-), z\right)\right] \tilde{N}(\mathrm{~d} s, \mathrm{~d} z)\right|^{2} \\
\left.\leq \frac{2 \epsilon}{\Gamma(\alpha)^{2}}\left\{\int_{0}^{t} \int_{Z}(t-s)^{2(\alpha-1)} E \mid F\left(s, X_{\epsilon}(s-), z\right)\right)-F\left(s, Z_{\epsilon}(s-), z\right)\right)\left.\right|^{2} v(\mathrm{~d} z) \mathrm{d} s \\
\left.\quad+\int_{0}^{t} \int_{Z}(t-s)^{2(\alpha-1)} E\left|F\left(s, Z_{\epsilon}(s-), z\right)-\bar{F}\left(Z_{\epsilon}(s-), z\right)\right|^{2} v(\mathrm{~d} z) \mathrm{d} s\right\},
\end{gathered}
$$

and so, by $\mathbf{H 1}$ and (3.4) in $\mathbf{H 3}$, we have

$$
\begin{aligned}
J_{3} \leq & \frac{2 K \epsilon}{\Gamma(\alpha)^{2}} \int_{0}^{t}(t-s)^{2(\alpha-1)} E\left|X_{\epsilon}(s-)-Z_{\epsilon}(s-)\right|^{2} \mathrm{~d} s \\
& \quad+\frac{2 \epsilon}{\Gamma(\alpha)^{2}} E \int_{0}^{t} \int_{Z}(t-s)^{2(\alpha-1)}\left|F(s, Z(s-), z)-\bar{F}\left(Z_{\epsilon}(s-), z\right)\right|^{2} v(\mathrm{~d} z) \mathrm{d} s \\
\leq & \frac{2 K \epsilon}{\Gamma(\alpha)^{2}} \int_{0}^{t}(t-s)^{2(\alpha-1)} E\left|X_{\epsilon}(s-)-Z_{\epsilon}(s-)\right|^{2} \mathrm{~d} s \\
& +\frac{2 K \epsilon}{\Gamma(\alpha)^{2}} t^{2 \alpha-1} \varphi_{3}(t)\left(1+E\left|Z_{\epsilon}(s-)\right|^{2}\right) .
\end{aligned}
$$

Therefore, from (3.7)-(3.9), Theorem 2.1, one can get

$$
\begin{aligned}
& E\left|X_{\epsilon}(t)-Z(t)\right|^{2} \\
\leq & \frac{6 t K \epsilon^{2}}{\Gamma(\alpha)^{2}} \int_{0}^{t}(t-s)^{2(\alpha-1)} E\left|X_{\epsilon}(s-)-Z_{\epsilon}(s-)\right|^{2} \mathrm{~d} s+\frac{6 K \epsilon^{2}}{\Gamma(\alpha)^{2}} t^{2 \alpha} \varphi_{1}(t)\left(1+E\left|Z_{\epsilon}(s-)\right|^{2}\right) \\
& \quad+\frac{6 K \epsilon}{\Gamma(\alpha)^{2}} \int_{0}^{t}(t-s)^{2(\alpha-1)} E\left|X_{\epsilon}(s-)-Z_{\epsilon}(s-)\right|^{2} \mathrm{~d} s+\frac{6 K \epsilon}{\Gamma(\alpha)^{2}} t^{2 \alpha-1} \varphi_{2}(t)\left(1+E\left|Z_{\epsilon}(s-)\right|^{2}\right) \\
& \quad+\frac{6 K \epsilon}{\Gamma(\alpha)^{2}} \int_{0}^{t}(t-s)^{2(\alpha-1)} E\left|X_{\epsilon}(s-)-Z_{\epsilon}(s-)\right|^{2} \mathrm{~d} s+\frac{6 K \epsilon}{\Gamma(\alpha)^{2}} t^{2 \alpha-1} \varphi_{3}(t)\left(1+E\left|Z_{\epsilon}(s-)\right|^{2}\right) \\
\leq & 6 \epsilon T^{2 \alpha-1}\left(\frac{C_{1} K \epsilon}{\Gamma(\alpha)^{2}} T+\frac{C_{2} K}{\Gamma(\alpha)^{2}}\right)
\end{aligned}
$$




$$
+6 \epsilon\left(\frac{K \epsilon}{\Gamma(\alpha)^{2}} T+\frac{2 K}{\Gamma(\alpha)^{2}}\right) \int_{0}^{t}(t-s)^{(2 \alpha-1)-1} E\left|X_{\epsilon}(s)-Z_{\epsilon}(s)\right|^{2} \mathrm{~d} s,
$$

where we have used the fact that $\left\{s: X_{\epsilon}(s) \neq X_{\epsilon}(s-)\right.$ or $\left.Z_{\epsilon}(s-) \neq Z_{\epsilon}(s)\right\}$ is countable. By setting $r_{1}=6\left(\frac{C_{1} K \epsilon}{\Gamma(\alpha)^{2}} T+\frac{C_{2} K}{\Gamma(\alpha)^{2}}\right)$ and $r_{2}=6\left(\frac{K \epsilon}{\Gamma(\alpha)^{2}} T+\frac{2 K}{\Gamma(\alpha)^{2}}\right)$, from Lemma 2.1, we have

$$
\begin{aligned}
E\left|X_{\epsilon}(t)-Z_{\epsilon}(t)\right|^{2} & \leq \epsilon T^{2 \alpha-1} r_{1}\left(1+\int_{0}^{t} \sum_{n=1}^{\infty} \frac{\left(r_{2} \epsilon \Gamma(2 \alpha-1)\right)^{n}}{\Gamma(2 n \alpha-n)}(t-s)^{n(2 \alpha-1)-1} \mathrm{~d} s\right) \\
& \leq \epsilon T^{2 \alpha-1} r_{1}\left(1+\sum_{n=1}^{\infty} \frac{\left(r_{2} \epsilon \Gamma(2 \alpha-1) T^{2 \alpha-1}\right)^{n}}{\Gamma(2 n \alpha-n+1)}\right) \\
& \leq \epsilon T^{2 \alpha-1} r_{1}\left(1+E_{2 \alpha-1,1}\left(r_{2} \epsilon \Gamma(2 \alpha-1) T^{2 \alpha-1}\right)\right) .
\end{aligned}
$$

By selecting $\beta \in(0,2 \alpha-1)$ and $L>0$, such that for every $t \in\left(0, L \epsilon^{\frac{-\beta}{2 \alpha-1}}\right) \subseteq[0, T]$, we obtain (3.6). This completes the proof.

\section{Acknowledgments}

Zhongkai Guo supported by NSF of China (Nos. 11526196, 11801575) and the Fundamental Research Funds for the Central Universities, South-Central University for Nationalities (Grant Number: CZY20014). Hongbo Fu is supported by NSF of China (Nos. 11826209, 11301403) and Natural Science Foundation of Hubei Province (No. 2018CFB688).

\section{References}

[1] Khasminskii R., On an averging principle for itô stochastic differential equations. Kibernetica, 4 (1968), 260-279.

[2] Bogoliubov N., Mitropolsky Y., Asymptotic methods in the theory of non-linear oscillations, Gordon and Breach Science Publishers, New York, 1961.

[3] Xu Y., Pei B. and Wu J., Stochastic averaging principle for differential equations with nonLipschitz coefficients driven by fractional Brownian motion. Stoch. Dyn., 17 (2017), 1750013.

[4] Dong Z., Sun X., Xiao H. and Zhai J., Averaging principle for one dimensional stochastic Burgers equation. J. Differ. Equ., 265 (2018), 4749-4797.

[5] Xu J., Liu J. and Miao Y., Strong averaging principle for two-time-scale SDEs with nonLipschitz coefficients. J. Math. Anal. Appl., 468 (2018), 116-140.

[6] Pei B., Xu Y. and Wu J., Stochastic averaging for stochastic differential equations driven by fractional Brownian motion and standard Brownian motion. Appl. Math. Lett., 100 (2020), 106006.

[7] Xu Y., Duan J. and Xu W., An averaging principle for stochastic dynamical sysytems with Lévy noise. Physica D, 240 (2011), 1395-1401.

[8] Conga N. D., Doanab T., Siegmundc S. and H. T. Tuan, On stable manifolds for planar fractional differential equations. Appl. Math. Comput., 226 (1) (2014), 157-168. 
[9] Wang Y., Xu J. and Kloeden P., Asymptotic behavior of stochastic lattice systems with a Caputo fractional time derivative. Nonlinear Analysis: Theory, Methods and Applications, 135 (2016), 205-222.

[10] Doan T., Kloeden P., Huong P. and Tuan H., Asymptotic separation between solutions of Caputo fractional stochastic differential equations. Stoch. Anal. Appl., 36 (4) (2018), 654-664.

[11] $\mathrm{Xu} \mathrm{W.,} \mathrm{Xu} \mathrm{W}$. and Zhang S., The averaging principle for stochastic differential equations with Caputo fractional derivative. Appl. Math. Lett., 93 (2019), 79-84.

[12] Xu W., Duan J. and Xu W., An averaging principle for fractional stochastic differential equations with Lévy noise. Chaos: An Interdisciplinary Journal of Nonlinear Science, 30 (8) (2020), 083126.

[13] Wang Y., Xu J. and Kloeden P. E., Asymptotic behavior of stochastic lattice systems with a Caputo fractional time derivative. Nonlinear Analysis: Theory, Methods Applications, 135 (2016), 205-222.

[14] Ye H., Gao J. and Ding Y., A generalized Gronwall inequality and its application to a fractional differential equation. J. Math. Anal. Appl., 328(2) (2007), 1075-1081.

[15] Wang W., Chen S., Guo Z. and Yan X., A note on the continuity for Caputo fractional stochastic differential equations. Chaos: An Interdisciplinary Journal of Nonlinear Science, 30(7) (2020), 073106. 